\title{
Metformin inhibits the proliferation of canine mammary gland tumor cells through the AMPK/AKT/mTOR signaling pathway in vitro
}

\author{
YUYING FAN $^{1 *}$, XIAOLI REN ${ }^{2 *}$, YINGXUE WANG $^{1}$, ENSHUANG XU $^{3}$, \\ SHUANG WANG ${ }^{1}$, RUIDONG GE ${ }^{1}$ and YUN LIU $^{1}$
}

\author{
${ }^{1}$ Department of Clinical Veterinary Medicine, College of Veterinary Medicine, Northeast Agricultural University, Harbin, \\ Heilongjiang $150000 ;{ }^{2}$ College of Veterinary Medicine, Henan University of Animal Husbandry and Economy, \\ Zhengzhou, Henan 450046; ${ }^{3}$ College of Animal Science and Veterinary Medicine, \\ Heilongjiang Bayi Agricultural University, Daqing, Heilongjiang 163000, P.R. China
}

Received October 8, 2020; Accepted June 14, 2021

DOI: $10.3892 / 01.2021 .13113$

\begin{abstract}
As an anti-diabetic drug, metformin has been demonstrated to exhibit antitumor effects. However, the mechanisms involved in decreasing tumor formation, including canine mammary gland tumors (CMGTs), are not well elucidated. The aim of the present study was to evaluate the ability of metformin to induce apoptosis and cell cycle arrest in CMGT cells, as well as identifying the pathways underlying these effects. Cell viability was assessed by Cell Counting Kit- 8 analysis following treating with metformin. Subsequently, apoptosis and cell cycle progression were assessed by flow cytometry, and the expression of associated proteins was examined. Expression levels of classical AMP-activated protein kinase (AMPK), protein kinase B (AKT), mechanistic target of rapamycin (mTOR) and eukaryotic translation initiation factor 4E-binding protein 1 (4E-BP1) were then investigated using western blot analysis. Metformin inhibited the proliferation of CHMm cells in a concentration-dependent manner. Specifically, metformin induced cell cycle arrest in the $G_{0} / G_{1}$ phases, accompanied by increased expression of p21 and p27, and decreased expression of cyclin D1 and cyclin-dependent kinase 4. Marked levels of apoptosis were observed in CHMm cells alongside the activation of caspase- 3 and cleavage of poly(ADP-ribose) polymerase. Also, the level of Bcl-2 was decreased, and that of Bax was increased. The expression of associated signaling molecules revealed that metformin
\end{abstract}

Correspondence to: Professor Yun Liu, Department of Clinical Veterinary Medicine, College of Veterinary Medicine, Northeast Agricultural University, 600 Changjiang Street, Harbin, Heilongjiang 150000, P.R. China

E-mail: abliuyun@yeah.net

*Contributed equally

Key words: metformin, canine mammary gland tumors, cell cycle, apoptosis, signal transduction markedly increased the phosphorylation of AMPK in CHMm cells, and decreased the levels of phosphorylated (p-)AKT, p-mTOR and p-4E-BP1, while Compound C reversed these changes. These findings demonstrated that metformin may be a potential therapeutic agent for CMGTs, acting via the AMPK/AKT/mTOR signaling pathway.

\section{Introduction}

Canine mammary gland tumors (CMGTs) are a serious threat to animal health, and are frequently seen in female dogs, $50 \%$ of which are malignant $(1,2)$. Research has shown that CMGTs share similarities with human breast cancer in clinical pathological features and pathogenesis, and therefore, CMGTs may offer a suitable model for the study of human breast cancer $(3,4)$.

Metformin (1,1-dimethylbiguanide) is used to treat type 2 diabetes, and due to its safety and tolerability, has been the first-line hypoglycemic drug since the 1950s (as recommended by the American Diabetes Association and the European Association for the Study of Diabetes) (5). It has previously been demonstrated that metformin also exerts non-hypoglycemic effects; epidemiological studies and meta-analyses have revealed that metformin decreases cancer risk and cancer-associated mortality compared with insulin or sulfonylureas in patients clinically treated for type 2 diabetes (6). Additionally, the anticancer effects of metformin have been demonstrated in human cancer cells and animal models of gastric, bladder and papillary thyroid cancers (7-9). Lengyel et al (10) and Chai et al (11) demonstrated that metformin markedly enhanced the sensitivity of tumor cells to chemotherapeutics, including gemcitabine and paclitaxel, suggesting its potential clinical use in overcoming the chemoresistance of tumor cells. Although studies investigating improvements in general immunity using metformin have seldom been reported, metformin may possess immune-mediated antitumor effects by increasing the number of $\mathrm{CD}^{+} \mathrm{T}$-cells, which are critical for protection against functional exhaustion in the tumor microenvironment $(12,13)$. 
The underlying molecular mechanisms involved in metformin-associated antitumor effects remain unknown. The AMP-activated protein kinase (AMPK)/mechanistic target of rapamycin (mTOR) signaling pathway is considered to be involved in the antitumor effect of metformin, similar to its hypoglycemic mechanism with regard to AMPK activation $(14,15)$. When cancer cells are treated with metformin, AMPK is activated, which inhibits the phosphorylation of mTOR, decreasing gene transcription, ribosome biogenesis and protein translation, and resulting in decreased cellular proliferation and division $(16,17)$. Although the phosphoinositide 3-kinase (PI3K)/protein kinase B (AKT) pathway serves an important function in chemoresistance, predictive/prognostic biomarkers and therapeutic applications for cancer, as well as being involved in maintaining glucose homeostasis with AMPK, the association between the AMPK/AKT/mTOR signaling pathway and the antitumor effects of metformin has rarely been studied in humans or canines $(18,19)$. The aim of the present study was to determine whether metformin exhibited antiproliferative effects and induced apoptosis and cell cycle arrest in CHMm cells, as well as identifying its association with the AMPK/AKT/mTOR signaling pathway, thus providing an experimental basis for further veterinary clinical application.

\section{Materials and methods}

Cell culture. The CMGT cell line, CHMm, was derived from pleural effusion of canine spontaneous mammary adenocarcinomas, and obtained from the Laboratory of Veterinary Surgery, Graduate School of Agriculture and Life Sciences, University of Tokyo (Tokyo, Japan) (20). The cells were cultured in DMEM with $10 \%$ fetal bovine serum, $1 \%$ penicillin-streptomycin and $1 \%$ glutamine at $37^{\circ} \mathrm{C}\left(5 \% \mathrm{CO}_{2}\right)$, and passaged in the logarithmic phase using 5\% Trypsin-EDTA.

Cell viability assessment. The Cell Counting Kit-8 (CCK-8; Dojindo Molecular Technologies, Inc.) was used to evaluate cell viability. CHMm cells were seeded into 96-well plates $\left(1,000\right.$ cells per well) and cultured at $37^{\circ} \mathrm{C}\left(5 \% \mathrm{CO}_{2}\right)$ until $80 \%$ confluent. The cells were then treated with $0,5,10,20$ and $40 \mathrm{mM}$ metformin (Sigma-Aldrich; Merck KGaA), and viability was assessed after $48 \mathrm{~h}$ incubation. After $1 \mathrm{~h}$ incubation with CCK- 8 reagent, the absorbance was measured at $450 \mathrm{~nm}$.

Apoptosis analysis. An Annexin V-fluorescein isothiocyanate (FITC) kit (Beyotime Institute of Biotechnology) was used to quantify apoptosis, according to the manufacturer's instructions. Cells were treated with or without $20 \mathrm{mM}$ metformin for $48 \mathrm{~h}$ at $37^{\circ} \mathrm{C}$ after serum starvation for $24 \mathrm{~h}$ (to facilitate drug absorption). The cells were harvested, washed with PBS, resuspended in Annexin V-FITC binding buffer and stained with Annexin V-FITC/propidium iodide (PI). The processed samples were examined by FACSCalibur flow cytometry (BD Biosciences) using FACSDiva software (version 6.1.3; BD Biosciences).

Cell cycle analysis. To assess the effects of metformin on the cell cycle of CMGT cells, CHMm cells were treated with or without $20 \mathrm{mM}$ metformin for $48 \mathrm{~h}$ after serum starvation for
$24 \mathrm{~h}$ to synchronize the cell cycle at the $\mathrm{G}_{0} / \mathrm{G}_{1}$ phase of the life cycle. Sample cells were rinsed with PBS and fixed with ice-cold $70 \%$ ethanol overnight in $4^{\circ} \mathrm{C}$, before being incubated with PI for $30 \mathrm{~min}$ at room temperature, as per the protocol of the Cell Cycle and Apoptosis kit (Beyotime Institute of Biotechnology). A flow cytometer and FACSDiva Version 6.1.3 software were used to assess cell cycle distribution.

Western blot analysis. CHMm cells were treated with 0 or $20 \mathrm{mM}$ metformin for $48 \mathrm{~h}$ after $2 \mathrm{~h}$ pretreatment with 0 or $10 \mu \mathrm{M}$ Compound C (CC; Selleck Chemicals). Cells were lysed at $4^{\circ}$ using Cell lysis buffer for Western and IP without inhibitors with $1 \mathrm{mM}$ PMSF (Beyotime Institute of Biotechnology) and the protein was collected. Protein determination was performed using the Enhanced BCA Protein Assay Kit (Beyotime Institute of Biotechnology). Equal amounts of protein $(30 \mu \mathrm{g} /$ lane) were separated using SDS-PAGE (10\% separation gel and $5 \%$ concentrated gel), and transferred to polyvinylidene difluoride membranes. The membranes were blocked with $5 \%$ fat-free milk including TBS-Tween $(0.1 \%)$ for $2 \mathrm{~h}$ at room temperature, and then incubated overnight at $4^{\circ} \mathrm{C}$ with the following primary antibodies(Albumin Bovine V, Beijing Solarbio Science \& Technology Co., Ltd.): Caspase-3 (cat. no. ab13847), poly(ADP-ribose) polymerase (PARP; cat. no. ab32071), cyclin D1 (cat. no. ab16663), cyclin-dependent kinase 4 (CDK4; cat. no. ab108357), p21 (cat. no. ab188224), p27 (cat. no. ab32034), AMPK (cat. no. ab3760), phosphorylated (p-)AMPK (Thr-183/172; cat. no. ab23875), AKT (cat. no. ab8805), p-AKT (Thr-308; cat. no. ab38449), mTOR (cat. no. ab2732), p-mTOR (Ser-2448; cat. no. ab84400) and $\beta$-actin (cat. no. ab6276) (all from Abcam), as well as 4E-BP1 (cat. no. bs-2559R) and p-4E-BP1(Thr-70; cat. no. bs-14550R) (both BIOSS). All primary antibodies were used at a 1:1,000 dilution in $5 \%$ fat-free milk with TBS-Tween $(0.1 \%)$. Detection was performed using HRP secondary antibodies (goat anti-rabbit; cat. no. ZB-2306 or goat-anti mouse; cat. no. ZB-2305; both Wanleibio Co., Ltd.; 1:3,000) for $2 \mathrm{~h}$ at room temperature, and visualized using enhanced chemiluminescence reagents (Tanon High-sig ECL Western Blotting Substrate; Tanon Science and Technology Co., Ltd.). Signals were quantified using Tanon GIS software (version 210.060.1000; Tanon Science and Technology Co., Ltd.).

Statistical analysis. All statistical analysis was performed using GraphPad Prism (version 5.0; GraphPad Software, Inc.), and the data are presented as the mean \pm SD of three experimental repeats. Comparisons among $\geq 3$ groups were analyzed using one-way ANOVA, and Tukey's test was used for further pairwise analysis. Unpaired Student's t-test was used to compare two groups. $\mathrm{P}<0.05$ was considered to indicate a statistically significant difference.

\section{Results}

Effects of metformin on CMGT cell viability. To determine the inhibitory effect of metformin on CMGT cell proliferation, cell viability was evaluated using the CCK-8 assay following treatment with $0,5,10,20$ or $40 \mathrm{mM}$ metformin. The results demonstrated that metformin reduced the viability and density of CHMm cells in a dose-dependent manner, suggesting that 

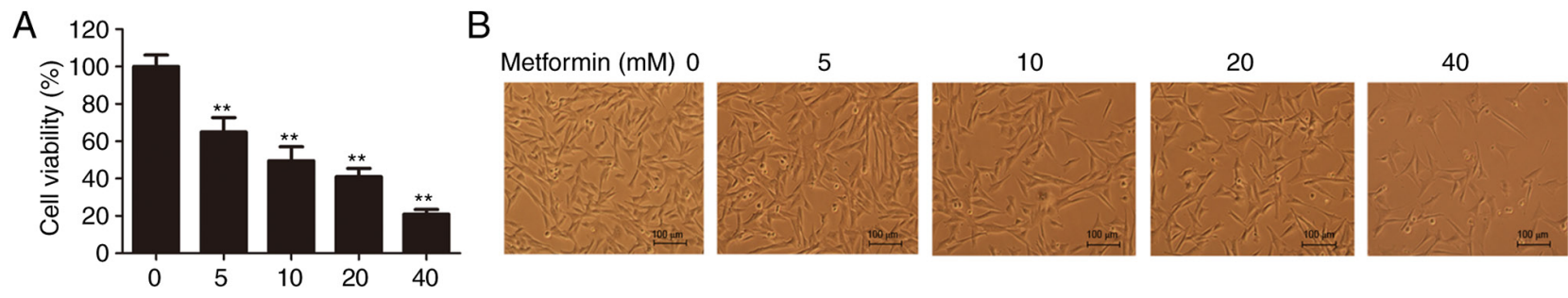

Figure 1. Antiproliferative effects of metformin on canine mammary gland tumor cells in vitro. (A) Cell viability in CHMm cells $48 \mathrm{~h}$ after treatment with the indicated concentrations of metformin. One-way ANOVA and Tukey's test were used for statistical analysis; ${ }^{* *} \mathrm{P}<0.01$ vs. control. (B) Cellular morphology was observed $48 \mathrm{~h}$ after treatment with various doses of metformin; magnification, x100. All data are presented as the mean $\pm \mathrm{SD}$.
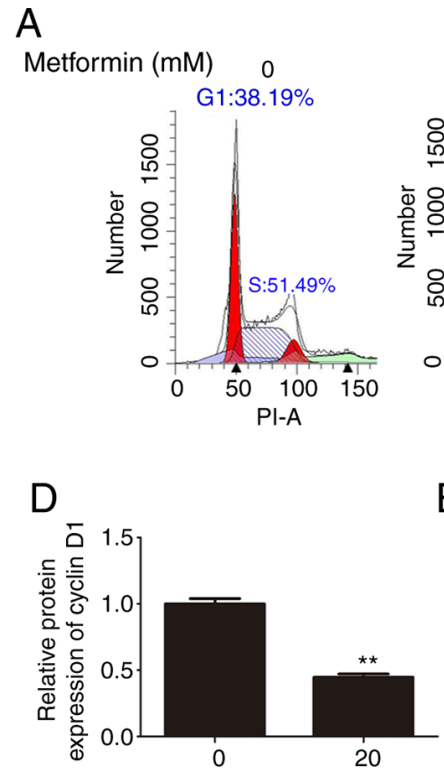

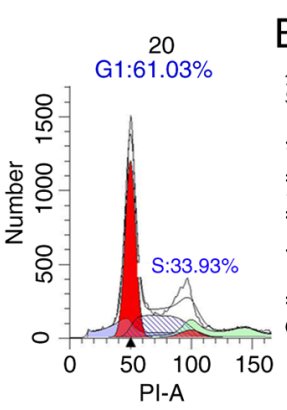

$\mathrm{E}$
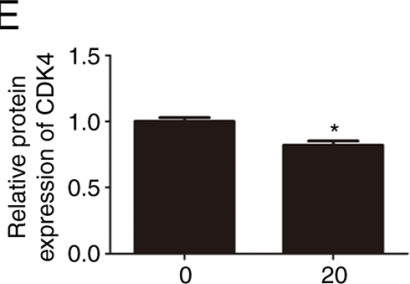

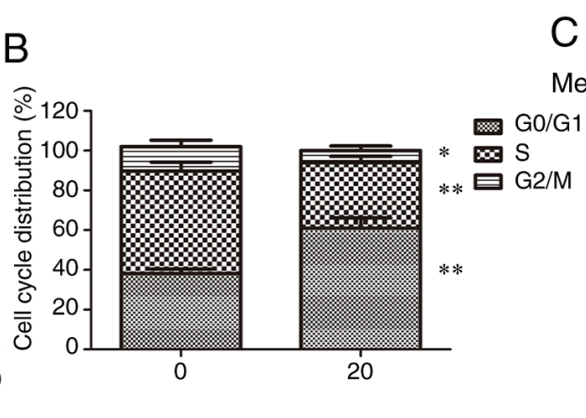

C

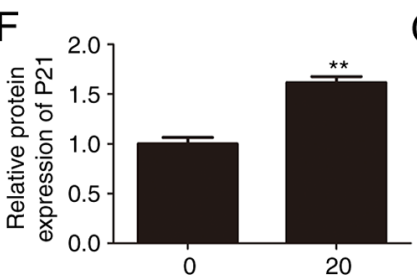

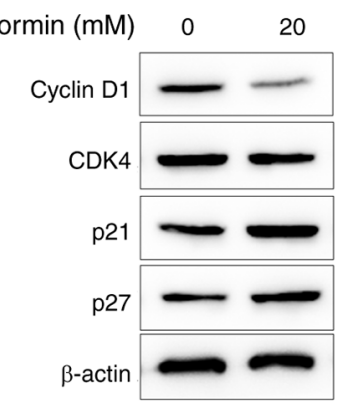

G

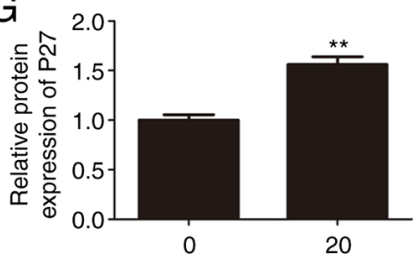

Figure 2. Effects of metformin on the canine mammary gland tumor cell cycle. (A) Percentages of $\mathrm{G}_{1}, \mathrm{~S}$ and $\mathrm{G}_{2}$ phase $\mathrm{CHMm}_{\mathrm{H}}$ cells $48 \mathrm{~h}$ after treatment with $20 \mathrm{mM}$ metformin, assessed using flow cytometry. (B) Proportions of CHMm cells in the $\mathrm{G}_{1}, \mathrm{~S}_{\text {and }} \mathrm{G}_{2}$ phases. (C) Cell cycle-associated proteins were analyzed using western blotting $48 \mathrm{~h}$ after treatment with $20 \mathrm{mM}$ metformin. Expression levels of (D) cyclin D1, (E) CDK4, (F) p21 and (G) p27 following treatment with or without $20 \mathrm{mM}$ metformin. ${ }^{*} \mathrm{P}<0.05$ and ${ }^{* *} \mathrm{P}<0.01$ vs. control. Unpaired Student's t-test was performed, and the results are presented as the mean $\pm \mathrm{SD}$. CDK4, cyclin dependent kinase 4.

metformin inhibits the proliferative ability of CMGT cells in vitro (Fig. $1 \mathrm{~A}$ and $\mathrm{B}$ ). As $20 \mathrm{mM}$ metformin reduced cell viability by $\sim 50 \%$, this concentration was the selected dose for subsequent experimentation.

Effects of metformin on the CMGT cell cycle. To further investigate whether the cell cycle is associated with metformin-induced reduction in CMGT cell proliferation, cell cycle distribution was flow cytometrically assessed following treatment with $20 \mathrm{mM}$ metformin. As presented in Fig. 2A and B, following metformin treatment, the percentage of cells in the $G_{1}$ and $S$ phases was markedly increased and decreased, respectively, under serum-deprived conditions. In addition, analysis of key $\mathrm{G}_{1}$ phase-modulating proteins supported that the cell cycle was arrested at the $\mathrm{G}_{0} / \mathrm{G}_{1}$, with decreased expression of cyclin D1 and CDK4, and increased expression of p21 and p27 (Fig. 2C-G). These results indicated that cell cycle arrest at the $G_{0} / G_{1}$ phase may be one of the underlying molecular mechanisms of the antitumor action of metformin.

Effects of metformin on apoptosis. To determine the effects of metformin on the apoptosis of CMGT cells, metformin-induced apoptosis was detected using Annexin V-FITC/PI staining and flow cytometry. Compared with the control group, $20 \mathrm{mM}$ metformin markedly increased the percentage of apoptotic CHMm cells under serum-deprived conditions (Fig. 3A and B). Additionally, metformin treatment resulted in marked activation of caspase-3, and PARP cleavage increased correspondingly (Fig. 3C-E), consistent with the quantitative results of flow cytometry. Also, metformin treatment significantly inhibited the expression of the anti-apoptotic protein $\mathrm{Bcl}-2$, and elevated the level of pro-apoptotic protein Bax (Fig. 3C, $\mathrm{F}$ and $\mathrm{G}$ ). Therefore, it was suggested that metformin induced the apoptosis of CHMm cells.

Effects on the AMPK/AKT/mTOR signaling pathway and its downstream targets. In order to determine the effects of metformin on AMPK/AKT/mTOR signaling, an AMPK inhibitor, CC, was selected, and expression of the associated signaling proteins was analyzed. CHMm cells were pretreated with or without $10 \mu \mathrm{M} \mathrm{CC}$ for $2 \mathrm{~h}$, and then treated with $20 \mathrm{mM}$ metformin for $48 \mathrm{~h}$. As illustrated in Fig. 4A, pretreatment with CC prevented the metformin-induced reduction in cell numbers. The results also demonstrated that metformin 

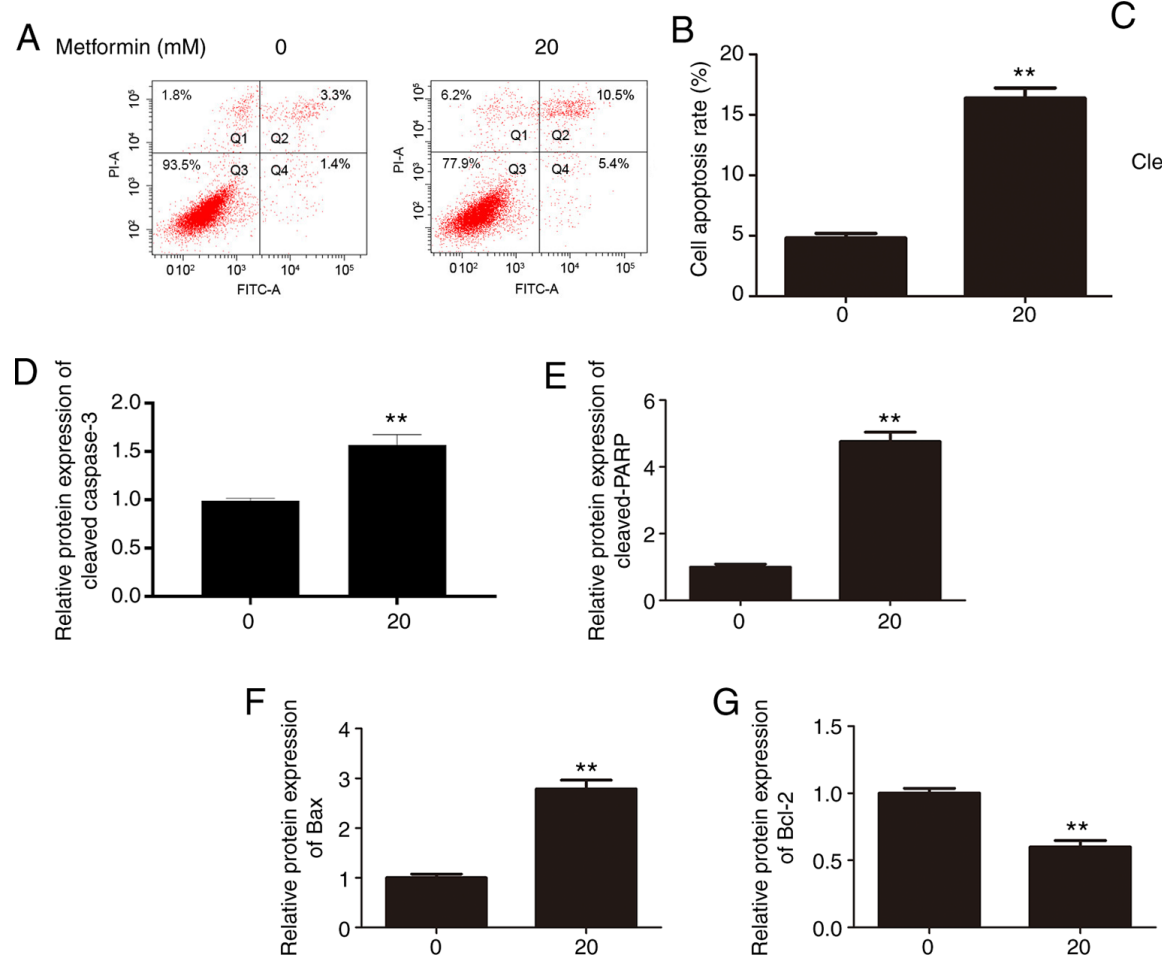

C

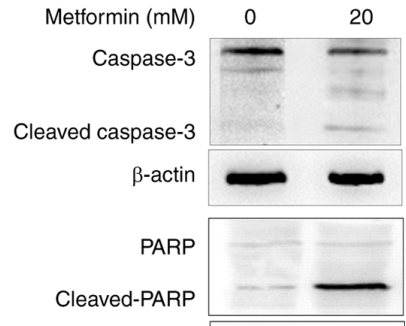

Figure 3. Effects of metformin on apoptosis in CHMm cells. (A) Flow cytometry was used to analyze apoptosis in CHMm cells $48 \mathrm{~h}$ after treatment with or without $20 \mathrm{mM}$ metformin. (B) Histograms present the percentage of apoptotic CHMm cells. Annexin V-FITC-positive cells (Q2 + Q4) were regarded as apoptotic. (C) Apoptosis-associated proteins were analyzed using western blot analysis $48 \mathrm{~h}$ after treatment with $20 \mathrm{mM}$ metformin. Expression levels of (D) cleaved-caspase-3, (E) cleaved-PARP, (F) Bax and (G) Bcl-2 following treatment with or without 20 mM metformin. Unpaired Student's t-test was performed, and the results are presented as the mean $\pm \mathrm{SD} .{ }^{* *} \mathrm{P}<0.01$ vs. control. PARP, poly(ADP-ribose) polymerase; FITC, fluorescein isothiocyanate.
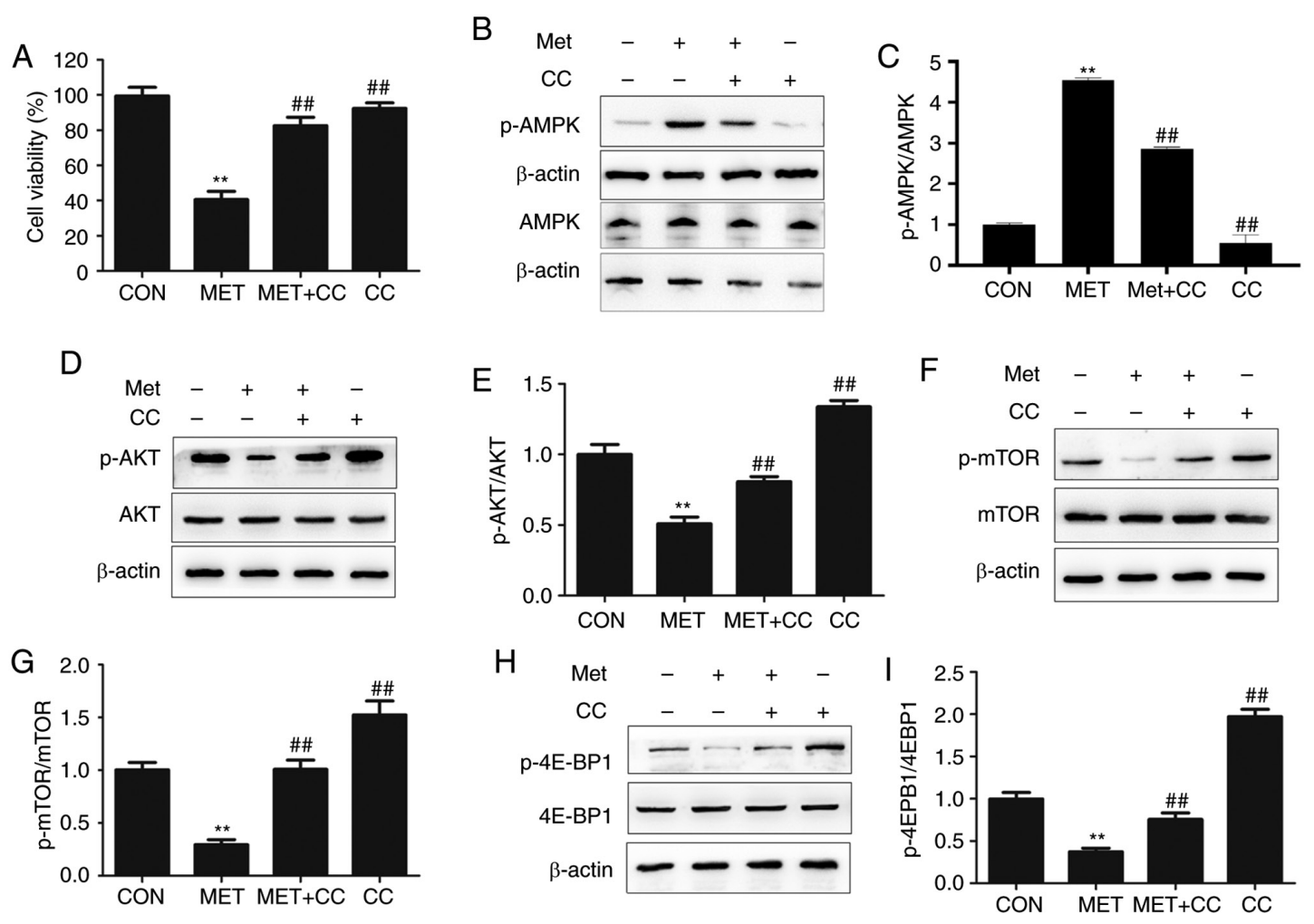

Figure 4. Effects of metformin on AMPK and downstream proteins. (A) Viability of CHMm cells was determined following treatment with metformin with or without the AMPK inhibitor CC at the 48-h time point. (B) Western blot analysis of p-AMPK and AMPK levels, (C) the p-AMPK to AMPK ratio, (D) p-AKT and AKT levels, (E) the p-AKT to AKT ratio, (F) p-mTOR and mTOR levels, (G) the p-mTOR to mTOR ratio, (H) p-4E-BP1 and 4E-BP1 levels, (I) and the p-4E-BP1 to 4E-BP1 ratio are presented following treatment with 0 or $20 \mathrm{mM}$ metformin, with or without CC. One-way ANOVA and Tukey's test were used for statistical analysis, and the results are presented as the mean $\pm \mathrm{SD} ;{ }^{* *} \mathrm{P}<0.01$ vs. control; ${ }^{\# \#} \mathrm{P}<0.01 \mathrm{vs}$. MET. $\beta \mathrm{AMPK}, \mathrm{AMP}$-activated protein kinase; AKT, protein kinase B: p-, phosphorylated; mTOR, mechanistic target of rapamycin; Met, metformin; CC, Compound C. 
increased the phosphorylation of AMPK in CHMm cells, as the ratio of p-AMPK to AMPK was increased (Fig. 4B and C). $\mathrm{CC}$ suppressed p-AMPK alone, and also significantly reversed the effect of metformin on AMPK phosphorylation compared with metformin treatment only. Furthermore, the level of p-AKT was significantly decreased following metformin treatment (Fig. 4D and E). As the key downstream components of the AMPK/AKT signaling pathway, p-mTOR and p-4E-BP1 exhibited decreased levels (Fig. 4F-I). The addition of CC also restored the levels of p-Akt, p-mTOR and p-4E-BP1 in metformin-treated CHMm cells (Fig. 4D, F and H). These results indicated that the molecular mechanism by which metformin reduces the proliferative capacity of CMGT cells may involve modulation of the AMPK/AKT/mTOR signaling pathway.

\section{Discussion}

The results of the present study demonstrated that metformin decreased the proliferation of CHMm cells in a dose-dependent manner in vitro. The antiproliferative effects of metformin treatment on canine tumor cells resembled that of previous studies on human tumor cells (7-9).

$\mathrm{G}_{0} / \mathrm{G}_{1}$ cell cycle arrest has been recognized as one mechanism underlying the antitumor effects of metformin, which has been confirmed in several cancer cell lines, and its regulation is dependent on the sequential activation and inactivation of cyclin-CDK-CD inhibitors (21-23). Previous studies have revealed that mitotic activity and cyclin D1 expression are associated: Canine mammary lesions that expressed cyclin D1 exhibited an increased proliferative ratio, and the opposite was true for cyclin D1-negative cell populations (24). In multiple myeloma cells, metformin treatment contributed to a concentration-dependent inhibition of proliferation through decreased cyclin D1 levels, associated with cell cycle arrest at the $\mathrm{G}_{0} / \mathrm{G}_{1}$ phase (25). Upregulation of CDK inhibitor 1 (p21Cip1) and CDK inhibitor $1 \mathrm{~B}$ (p27Kip1), as well as downregulation of cyclin D1, were involved in metformin-induced inhibition of tumor growth and cell cycle arrest in esophageal squamous cell carcinomas (26). The results of the present study demonstrated that metformin may arrest CMGT cells in the $\mathrm{G}_{0} / \mathrm{G}_{1}$ phases due to the increased expression of $\mathrm{p} 27$ and $\mathrm{p} 21$, and the decreased expression of CDK4 and cyclin D1. Thus, downregulation of cyclin D1 may contribute to cell cycle arrest and be vital for the anticancer effects of metformin in CMGT cells.

There may be different mechanisms that regulate cancer cell apoptosis. As a programmed cell death process, apoptosis can be induced by the mitochondrial, death receptor-mediated pathway and endoplasmic reticulum pathway (27). Apoptosis is associated with a variety of cellular proteins, including caspase-3, which is essential for cellular repair and apoptotic cell death (28). The active form of caspase-3 proteolytically cleaves PARP, which is involved in DNA repair and replication, cellular proliferation and differentiation, thus caspase- 3 is regarded as a marker of apoptosis (29). Previous studies have suggested that differences in induced apoptosis may be linked to the species and genotype of cancer cells; for example, metformin-induced apoptosis was associated with the B-cell lymphoma 2 family in epithelial ovarian cancer (30).
Metformin also impaired the proliferation p53-deficient colon tumor cells $(31,32)$. However, in multiple myeloma, metformin inhibited cellular proliferation through $\mathrm{G}_{0} / \mathrm{G}_{1}$ phase arrest rather than apoptosis (25). Metformin was determined to increase apoptotic cell death and significantly increase cleaved caspase- 3 and cleaved PARP levels, thus metformin may have play a therapeutic role in rectal cancer (33). The present study revealed that metformin induced apoptosis in CHMm cells. The results demonstrated that $\mathrm{CHMm}$ cells treated with metformin showed increased caspase-3 activity and cleavage of PARP, supporting the association between PARP cleavage and caspase activation during apoptosis. The results also showed that following metformin treatment, the anti-apoptotic protein Bcl-2 was significantly downregulated, while pro-apoptotic Bax was markedly upregulated, in CHMm cells.

It has been demonstrated that distinct subtypes of CMGT cells are inhibited by metformin to various degrees, as a result of distinct mechanisms, e.g. in an AMPK-independent or -dependent manner (34). A study showed that treatment with $20 \mathrm{mM}$ metformin resulted in the upregulation of $\mathrm{p}$-AMPK and the downregulation of $\mathrm{p}-\mathrm{AKT}$, which was associated with $\mathrm{G}_{0} / \mathrm{G}_{1}$ phase cell cycle arrest, apoptotic and non-apoptotic death in MCF-7 breast cancer cells (35). Similarly, metformin was found to induce apoptosis and inhibit migration by activating $\mathrm{AMPK} / \mathrm{p} 53$ and suppressing PI3K/AKT signaling in human cervical cancer cells (36). In an experiment involving a xenograft murine model, metformin was demonstrated to enhance the anti-cancer effect of cisplatin in meningioma by upregulating AMPK and downregulating mTOR signaling in vivo (37). The present study investigated the effects of metformin on the AMPK signaling pathway in CHMm cells. The results revealed that metformin treatment increased p-AMPK levels, and that CC attenuated the effects of metformin on AMPK signaling.

It has also been reported that metformin inhibited the proliferation of human breast cancer cells by decreasing AKT activation and repressing associated downstream molecules (38). In the present study, AKT phosphorylation was inhibited by metformin treatment and reversed by CC in CHMm cells, demonstrating that the AKT signaling pathway is involved in the antitumor effects of metformin. However, the exact molecular mechanism underlying metformin-induced inhibition of the AKT pathway remains unclear.

mTOR is an essential mediator of growth signals regulated by the AMPK/AKT signaling pathway; moreover, activating AMPK and inhibiting AKT may restrain the activity of mTOR and thereby exert an antitumor effect $(39,40)$. A study indicated that metformin inhibits the proliferation of myeloma cells through $\mathrm{G}_{0} / \mathrm{G}_{1}$ phase cell cycle arrest, potentially by repressing the mTORC1 and mTORC2 pathways (which are mediated by AMPK activation) (25). A previous immunohistochemical study have suggested that p-mTOR was not present in normal canine mammary tissue, but expressed in the cytoplasm of canine mammary carcinoma cells, which indicated that p-mTOR was involved in mammary carcinogenesis in dogs (41). Furthermore, inhibition of the mTOR signaling pathway in canine mammary carcinoma cells demonstrated significant anticancer activity in vitro, identifying it as a promising therapeutic target (42). In the current study, metformin treatment decreased the phosphorylation of mTOR and 
4E-BP1, which was increased following treatment with CC, confirming that the mTOR signaling pathway was inhibited in $\mathrm{CHMm}$ cells. Collectively, the present study suggested a novel inhibitory function of metformin in CMGTs through its action on the AMPK/AKT/mTOR signaling pathway.

In conclusion, the results of the present study demonstrated that metformin was able to inhibit the proliferation of CMGT cells, potentially through cell cycle arrest, induced apoptosis, activation of AMPK and suppression of AKT/mTOR signaling pathways. These results suggest that metformin may be a potential chemotherapeutic agent in the treatment of canine mammary gland tumors, and may offer insights into potential avenues of human breast cancer research.

\section{Acknowledgements}

Not applicable.

\section{Funding}

The present study was supported by grants from the National Natural Science Foundation of China (grant no. 31672617), the National Key Research Projects (grant no. 2016YFD0501008), the Initial Scientific Doctoral Research Foundation in Henan University of Animal Husbandry and Economy (grant no. 2019HNUAHEDF025), and Key projects of Henan Province Colleges and Universities (grant no. 20B230004).

\section{Availability of data and materials}

The datasets used and/or analyzed during the current study are available from the corresponding author on reasonable request.

\section{Authors' contributions}

YL conceived the project and designed the experiments. YF and XR wrote and revised the manuscript. YF, XR, EX and SW analyzed the data. YW, XR, YF and RG conducted the experiments. YL and YW confirmed the authenticity of all the raw data. All authors have read and approved the final manuscript.

\section{Ethics approval and consent to participate}

Not applicable.

\section{Patient consent for publication}

Not applicable.

\section{Competing interests}

The authors declare that they have no competing interests.

\section{References}

1. Vascellari M, Capello K, Carminato A, Zanardello C, Baioni E and Mutinelli F: Incidence of mammary tumors in the canine population living in the Veneto region (Northeastern Italy) Risk factors and similarities to human breast cancer. Prev Vet Med 126: 183-189, 2016.
2. Bird RC, DeInnocentes P, Church Bird AE, Lutful Kabir FM, Martinez-Romero EG, Smith AN and Smith BF: Autologous hybrid cell fusion vaccine in a spontaneous intermediate model of breast carcinoma. J Vet Sci 20: e48, 2019.

3. Mohammed SI, Meloni GB, Pinna Parpaglia ML, Marras V, Burrai GP, Meloni F, Pirino S and Antuofermo E: Mammography and ultrasound imaging of preinvasive and invasive canine spontaneous mammary cancer and their similarities to human breast cancer. Cancer Prev Res (Phila) 4: 1790-1798, 2011.

4. Abdelmegeed SM and Mohammed S: Canine mammary tumors as a model for human disease. Oncol Lett 15: 8195-8205, 2018.

5. Sanchez-Rangel E and Inzucchi SE: Metformin: Clinical use in type 2 diabetes. Diabetologia 60: 1586-1593, 2017.

6. Baur DM, Klotsche J, Hamnvik OP, Sievers C, Pieper L, Wittchen HU, Stalla GK, Schmid RM, Kales SN and Mantzoros CS: Type 2 diabetes mellitus and medications for type 2 diabetes mellitus are associated with risk for and mortality from cancer in a German primary care cohort. Metabolism 60: 1363-1371, 2011.

7. Kato K, Gong J, Iwama H, Kitanaka A, Tani J, Miyoshi H, Nomura K, Mimura S, Kobayashi M, Aritomo Y, et al: The antidiabetic drug metformin inhibits gastric cancer cell proliferation in vitro and in vivo. Mol Cancer Ther 11: 549-560, 2012.

8. Cho SW, Yi KH, Han SK, Sun HJ, Kim YA, Oh BC, Park YJ and Park DJ: Therapeutic potential of metformin in papillary thyroid cancer in vitro and in vivo. Mol Cell Endocrinol 393: 24-29, 2014

9. Zhang T, Guo P, Zhang Y, Xiong H, Yu X, Xu S, Wang X, He D and Jin X: The antidiabetic drug metformin inhibits the proliferation of bladder cancer cells in vitro and in vivo. Int J Mol Sci 14: 24603-24618, 2013.

10. Lengyel E, Litchfield LM, Mitra AK, Nieman KM, Mukherjee A, Zhang Y, Johnson A, Bradaric M, Lee W and Romero IL: Metformin inhibits ovarian cancer growth and increases sensitivity to paclitaxel in mouse models. Am J Obstet Gynecol 212: 479.e1-479.e10, 2015.

11. Chai X, Chu H, Yang X, Meng Y, Shi P and Gou S: Metformin increases sensitivity of pancreatic cancer cells to gemcitabine by reducing $\mathrm{CD}_{133^{+}}$cell populations and suppressing ERK/P70S6K signaling. Sci Rep 5: 14404, 2015.

12. Eikawa S, Nishida M, Mizukami S, Yamazaki C, Nakayama E and Udono H: Immune-mediated antitumor effect by type 2 diabetes drug, metformin. Proc Natl Acad Sci USA 112: 1809-1814, 2015.

13. Watanabe M, Yamamoto H, Eikawa S, Shien K, Shien T, Soh J, Hotta K, Wada J, Hinotsu S, Fujiwara T, et al: Study about the efficacy of metformin to immune function in cancer patients. Acta Med Okayama 70: 327-330, 2016.

14. Shi WY, Xiao D, Wang L, Dong LH, Yan ZX, Shen ZX, Chen SJ, Chen Y and Zhao WL: Therapeutic metformin/AMPK activation blocked lymphoma cell growth via inhibition of mTOR pathway and induction of autophagy. Cell Death Dis 3: e275, 2012.

15. Han G,Gong H,Wang Y,GuoS andLiu K: AMPK/mTOR-mediated inhibition of survivin partly contributes to metformin-induced apoptosis in human gastric cancer cell. Cancer Biol Ther 16: 77-87, 2015.

16. Kimball SR: Interaction between the AMP-activated protein kinase and mTOR signaling pathways. Med Sci Sports Exerc 38: 1958-1964, 2006.

17. Yue W, Yang CS, DiPaola RS and Tan XL: Repurposing of metformin and aspirin by targeting AMPK-mTOR and inflammation for pancreatic cancer prevention and treatment. Cancer Prev Res (Phila) 7: 388-397, 2014.

18. Bahrami A, Khazaei M, Hasanzadeh M, ShahidSales S, Joudi Mashhad M, Farazestanian M, Sadeghnia HR, Rezayi M, Maftouh M, Hassanian SM, et al: Therapeutic potential of targeting PI3K/AKT pathway in treatment of colorectal cancer: rational and progress. J Cell Biochem 119: 2460-2469, 2018.

19. Schultze SM, Hemmings BA, Niessen M and Tschopp O: PI3K/AKT, MAPK and AMPK signalling: Protein kinases in glucose homeostasis. Expert Rev Mol Med 14: e1, 2012.

20. Uyama R, Nakagawa T, Hong SH, Mochizuki M, Nishimura R and Sasaki N: Establishment of four pairs of canine mammary tumour cell lines derived from primary and metastatic origin and their E-cadherin expression. Vet Comp Oncol 4: 104-113, 2006.

21. Jin DH, Kim Y, Lee BB, Han J, Kim HK, Shim YM and Kim DH: Metformin induces cell cycle arrest at the G1 phase through E2F8 suppression in lung cancer cells. Oncotarget 8: 101509-101519, 2017.

22. Xie W, Wang L, Sheng H, Qiu J, Zhang D, Zhang L, Yang F, Tang D and Zhang K: Metformin induces growth inhibition and cell cycle arrest by upregulating MicroRNA34a in renal cancer cells. Med Sci Monit 23: 29-37, 2017. 
23. Canavese M, Santo L and Raje N: Cyclin dependent kinases in cancer: Potential for therapeutic intervention. Cancer Biol Ther 13: 451-457, 2012.

24. Sfacteria A, Bertani C, Costantino G, Del Bue M, Paiardini M, Cervasi B, Piedimonte A and De Vico G: Cyclin D1 expression in pre-cancerous and cancerous lesions of the canine mammary gland. J Comp Pathol 128: 245-251, 2003.

25. Wang Y, Xu W, Yan Z, Zhao W, Mi J, Li J and Yan H: Metformin induces autophagy and G0/G1 phase cell cycle arrest in myeloma by targeting the AMPK/mTORC1 and mTORC 2 pathways. J Exp Clin Cancer Res 37: 63, 2018.

26. Cai X, Hu X, Tan X, Cheng W, Wang Q, Chen X, Guan Y, Chen C and Jing X: Metformin induced AMPK activation, G0/G1 phase cell cycle arrest and the inhibition of growth of esophageal squamous cell carcinomas in vitro and in vivo. PLoS One 10: e0133349, 2015.

27. Gao S, Tan H, Zhu N, Gao H, Lv C, Gang J and Ji Y: Oridonin induces apoptosis through the mitochondrial pathway in human gastric cancer SGC-7901 cells. Int J Oncol 48: 2453-2460, 2016.

28. Xiong ZS, Gong SF, Si W, Jiang T, Li QL, Wang TJ, Wang WJ, Wu RY and Jiang K: Effect of metformin on cell proliferation, apoptosis, migration and invasion in A172 glioma cells and its mechanisms. Mol Med Rep 20: 887-894, 2019.

29. Sergeeva TF, Shirmanova MV, Zlobovskaya OA, Gavrina AI, Dudenkova VV, Lukina MM, Lukyanov KA and Zagaynova EV: Relationship between intracellular $\mathrm{pH}$, metabolic co-factors and caspase-3 activation in cancer cells during apoptosis. Biochim Biophys Acta Mol Cell Res 1864: 604-611, 2017.

30. Yasmeen A, Beauchamp MC, Piura E, Segal E, Pollak M and Gotlieb WH: Induction of apoptosis by metformin in epithelial ovarian cancer: Involvement of the $\mathrm{Bcl}-2$ family proteins. Gynecol Oncol 121: 492-498, 2011.

31. Li X, Li B, Ni Z, Zhou P, Wang B, He J, Xiong H, Yang F, Wu Y, Lyu X, et al: Metformin synergizes with BCL-XL/BCL-2 inhibitor ABT-263 to induce apoptosis specifically in p53-defective cancer cells. Mol Cancer Ther 16: 1806-1818, 2017.

32. Buzzai M, Jones RG, Amaravadi RK, Lum JJ, DeBerardinis RJ, Zhao F, Viollet B and Thompson CB: Systemic treatment with the antidiabetic drug metformin selectively impairs p53-deficient tumor cell growth. Cancer Res 67: 6745-6752, 2007.
33. Park JH, Kim YH, Park EH, Lee SJ, Kim H, Kim A, Lee SB, Shim S, Jang H, Myung JK, et al: Effects of metformin and phenformin on apoptosis and epithelial-mesenchymal transition in chemoresistant rectal cancer. Cancer Sci 110: 2834-2845, 2019.

34. Saeki K, Watanabe M, Tsuboi M, Sugano S, Yoshitake R, Tanaka Y, Ong SM, Saito T, Matsumoto K, Fujita N, et al: Anti-tumour effect of metformin in canine mammary gland tumour cells. Vet J 205: 297-304, 2015.

35. Queiroz EA, Puukila S, Eichler R, Sampaio SC, Forsyth HL, Lees SJ, Barbosa AM, Dekker RF, Fortes ZB and Khaper N: Metformin induces apoptosis and cell cycle arrest mediated by oxidative stress, AMPK and FOXO3a in MCF-7 breast cancer cells. PLoS One 9: e98207, 2014.

36. Chen YH, Yang SF, Yang CK, Tsai HD, Chen TH, Chou MC and Hsiao YH: Metformin induces apoptosis and inhibits migration by activating the AMPK/p53 axis and suppressing PI3K/AKT signaling in human cervical cancer cells. Mol Med Rep 23: 23, 2021.

37. Guo L, Cui J, Wang H, Medina R and Lin Y: Metformin enhances anti-cancer effect of cisplatin in meningioma through AMPK-mTOR signaling pathways. Mol Ther Oncolytics 20: 119-131, 2020.

38. Zakikhani M, Blouin MJ, Piura E and Pollak MN: Metformin and rapamycin have distinct effects on the AKT pathway and proliferation in breast cancer cells. Breast Cancer Res Treat 123: 271-279, 2010.

39. Morgensztern D and McLeod HL: PI3K/Akt/mTOR pathway as a target for cancer therapy. Anticancer Drugs 16: 797-803, 2005.

40. Dey N, De P and Leyland-Jones B: PI3K-AKT-mTOR inhibitors in breast cancers: From tumor cell signaling to clinical trials. Pharmacol Ther 175: 91-106, 2017.

41. Delgado L, Gärtner F and Dias Pereira P: Activation of mammalian target of rapamycin in canine mammary carcinomas: An immunohistochemical study. J Comp Pathol 152: 138-144, 2015.

42. Chen YT, Tan KA, Pang LY and Argyle DJ: The class I PI3K/Akt pathway is critical for cancer cell survival in dogs and offers an opportunity for therapeutic intervention. BMC Vet Res 8: 73, 2012.

This work is licensed under a Creative Commons Attribution-NonCommercial-NoDerivatives 4.0 International (CC BY-NC-ND 4.0) License. 